International Journal of Pure and Applied Mathematics

Volume 93 No. 3 2014, 339-350

ISSN: 1311-8080 (printed version); ISSN: 1314-3395 (on-line version)

url: http://www.ijpam.eu

doi: http://dx.doi.org/10.12732/ijpam.v93i3.4

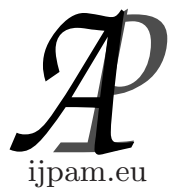

\title{
AFFINE MAPPINGS ON SIMPLICES
}

\author{
Malinee Chaiya ${ }^{1}$, Somjate Chaiya ${ }^{2} \S$ \\ ${ }^{1,2}$ Department of Mathematics \\ Faculty of Science \\ Silpakorn University \\ Nakorn Pathom, 73000, THAILAND
}

\begin{abstract}
In this paper, we study affine mappings on domains in $\mathbb{R}^{3}$ that are unions of simplices. Let $f_{i}$ be an affine mapping of the form $f_{i}(x)=A_{i} x$, where $A_{i}$ is a $3 \times 3$ transformation matrix, on a simplex $Q_{i}$. We establish the condition of these matrices $A_{i}$ in order to obtain a continuous piecewise affine mapping $f$ on the domain $Q$ that is the union of simplices $Q_{i}$ such that $f \mid Q_{i}=f_{i}$.
\end{abstract}

AMS Subject Classification: 14R99, 15A23

Key Words: affine mapping, simplex

\section{Introduction}

An affine mapping consists of a linear transformation followed by a translation. An affine mapping $f$ in $\mathbb{R}^{n}$ is of the form

$$
f(x)=A x+b
$$

where $A$ is an $n \times n$ transformation matrix and $b$ is a vector in $\mathbb{R}^{n}$. Geometrically, an affine mapping in the Euclidean space preserves the collinearity relation between points and the ratio of distances along a straight line.

Received: November 16, 2013

(C) 2014 Academic Publications, Ltd.

$\S$ Correspondence author url: www.acadpubl.eu 
Let $Q$ be a domain in $\mathbb{R}^{3}$ that is a union of simplices $Q_{i}$ whose interiors are disjoint. Consider an affine mapping on each simplex $Q_{i}$ of the form $f_{i}(x)=$ $A_{i} x$, where $A_{i}$ is a $3 \times 3$ transformation matrix. We want to study the condition of these matrices $A_{i}$ in order to obtain a continuous piecewise affine mapping $f$ on the domain $Q$ such that $f \mid Q_{i}=f_{i}$. By the Singular value decomposition theorem, each $A_{i}$ can be written as $U_{i} D_{i} V_{i}^{T}$, where $U_{i}$ and $V_{i}$ are orthogonal matrices and $D_{i}$ is a non-singular diagonal matrix. Notice that $A_{i}^{T} A_{i}=V_{i} D_{i}^{2} V_{i}^{T}$ is independent from $U_{i}$. To obtain the necessary and sufficient conditions for $A_{i}$ so that $f$ is continuous, we will investigate the conditions for $D_{i}$ and $V_{i}$ that affirm the existence of $U_{i}$ such that the matrix $A_{i}=U_{i} D_{i} V_{i}^{T}$ satisfies the desired property.

\section{Two Simplices Sharing a Common Face}

We study first the simplest case when the domain $Q$ is a union of two simplices sharing a common face. Let $Q_{1}$ and $Q_{2}$ be simplices sharing a common face which lies in a plane $T$. We call the plane $T$ the common plane. For convenience, we may assume that the origin 0 is a common vertex of $Q_{1}$ and $Q_{2}$.

Let $f_{i}(x)=A_{i} x$ be an affine mapping on $Q_{i}$ and $U_{i} D_{i} V_{i}^{T}$ be the decomposition of $A_{i}$. To obtain a continuous piecewise affine mapping $f$ on the domain $Q=Q_{1} \cup Q_{2}$ such that $f \mid Q_{i}=f_{i}, f_{1}$ and $f_{2}$ must satisfy $f_{1}(x)=f_{2}(x)$ for all $x$ on the plane $T$. That is, for all $x$ on $T$

$$
U_{1} D_{1} V_{1}^{T} x=U_{2} D_{2} V_{2}^{T} x .
$$

If $A_{1}$ is given, we want to know whether there exists a matrix $A_{2}$ that satisfies (1). In [1], Sinthaveelert gave the necessary and sufficient condition for the existence of $A_{2}$.

Theorem 1. Suppose that $U_{1}, D_{1}, D_{2}, V_{1}$, and $V_{2}$ are given. There exists an orthogonal matrix $U_{2}$ satisfying (1) for all $x \in T$ if, and only if, for all $h_{1}, h_{2} \in T$

$$
h_{2}^{T} V_{2} D_{2}^{2} V_{2}^{T} h_{1}=h_{2}^{T} V_{1} D_{1}^{2} V_{1}^{T} h_{1} .
$$

Proof. We suppose first that there exists a matrix $U_{2}$ satisfying the requirement. Therefore, for any $h_{1}, h_{2} \in T$,

$$
\left(U_{2} D_{2} V_{2}^{T} h_{2}\right)^{T} U_{2} D_{2} V_{2}^{T} h_{1}=\left(U_{1} D_{1} V_{1}^{T} h_{2}\right)^{T} U_{1} D_{1} V_{1}^{T} h_{1},
$$

which gives $h_{2}^{T} V_{2} D_{2}^{2} V_{2}^{T} h_{1}=h_{2}^{T} V_{1} D_{1}^{2} V_{1}^{T} h_{1}$ because $U_{i} U_{i}^{T}=I=U_{i}^{T} U_{i}$. 
Next suppose that $h_{2}^{T} V_{2} D_{2}^{2} V_{2}^{T} h_{1}=h_{2}^{T} V_{1} D_{1}^{2} V_{1}^{T} h_{1}$ for all $h_{1}, h_{2} \in T$. We want to show that we can find an orthogonal matrix $U_{2}$ that satisfies (1).

Let $t_{1}$ and $t_{2}$ be vectors in $\mathbb{R}^{3}$ such that $\operatorname{span}\left\{t_{1}, t_{2}\right\}=T$. Set $x=D_{2} V_{2}^{T} t_{1}$ and $y=D_{2} V_{2}^{T} t_{2}$. Notice that $\{x, y, x \times y\}$ is a basis for $\mathbb{R}^{3}$ and $\operatorname{span}\{x, y\}=$ $D_{2} V_{2}^{T} T$. To satisfy (1), we need to define $U_{2}$ so that for all $z \in D_{2} V_{2}^{T} T$

$$
U_{2} z=U_{1} D_{1} V_{1}^{T} V_{2} D_{2}^{-1} z .
$$

Indeed we need only the condition that

$$
U_{2} x=U_{1} D_{1} V_{1}^{T} V_{2} D_{2}^{-1} x \quad \text { and } \quad U_{2} y=U_{1} D_{1} V_{1}^{T} V_{2} D_{2}^{-1} y .
$$

Let $r=U_{1} D_{1} V_{1}^{T} V_{2} D_{2}^{-1} x, s=U_{1} D_{1} V_{1}^{T} V_{2} D_{2}^{-1} y$, and let $\gamma=\frac{ \pm|x \times y|}{|r \times s|}$. Then let

$$
U_{2}=\left[\begin{array}{lll}
r & s & \gamma(r \times s)
\end{array}\right]\left[\begin{array}{lll}
x & y & (x \times y)
\end{array}\right]^{-1} .
$$

It is easy to see that $U_{2} x=r$ and $U_{2} y=s$, hence $U_{2}$ fulfills the first part of the desired condition. We still have to verify that $U_{2}$ is orthogonal. Since $\{x, y, x \times y\}$ is a basis for $\mathbb{R}^{3}$, any vectors $a$ and $b$ in $\mathbb{R}^{3}$ can be represented as linear combinations of $x, y$, and $x \times y$. For instance, let $a=a_{1} x+a_{2} y+$ $a_{3}(x \times y)$ and $b=b_{1} x+b_{2} y+b_{3}(x \times y)$. Note that we have

$$
\begin{aligned}
r^{T} r & =x^{T}\left(D_{2}^{-1}\right)^{T} V_{2}^{T} V_{1} D_{1}^{2} V_{1}^{T} V_{2} D_{2}^{-1} x \\
& =x^{T}\left(D_{2}^{-1}\right)^{T} V_{2}^{T} V_{2} D_{2}^{2} V_{2}^{T} V_{2} D_{2}^{-1} x=x^{T} x .
\end{aligned}
$$

Similarly, $r^{T} s=x^{T} y, s^{T} s=y^{T} y$, and $s^{T} r=y^{T} x$. Therefore

$$
\begin{aligned}
a \cdot b= & \left(a_{1} x+a_{2} y+a_{3}(x \times y)\right) \cdot\left(b_{1} x+b_{2} y+b_{3}(x \times y)\right) \\
= & a_{1} b_{1}\left(x^{T} x\right)+a_{2} b_{2}\left(y^{T} y\right)+a_{3} b_{3}\left((x \times y)^{T}(x \times y)\right) \\
& +a_{1} b_{2}\left(x^{T} y\right)+a_{2} b_{1}\left(y^{T} x\right) \\
= & a_{1} b_{1}\left(r^{T} r\right)+a_{2} b_{2}\left(s^{T} s\right)+a_{3} b_{3} \gamma^{2}\left((r \times s)^{T}(r \times s)\right) \\
& +a_{1} b_{2}\left(r^{T} s\right)+a_{2} b_{1}\left(s^{T} r\right) \\
= & \left(a_{1} r+a_{2} s+a_{3} \gamma(r \times s)\right) \cdot\left(b_{1} r+b_{2} s+b_{3} \gamma(r \times s)\right) \\
= & U_{2} a \cdot U_{2} b .
\end{aligned}
$$

Hence, $U_{2}$ preserves dot products and then it also preserves lengths. Thus $U_{2}$ is orthogonal, which completes the proof.

Remark that the existence of $U_{2}$ is independent from $U_{1}$, but to find such $U_{2}$ it definitely involves $U_{1}$. 


\section{Four Simplices Sharing a Common Edge}

In this section, we turn our attention to a domain that is a union of four simplices. For the case when a domain is a union of three simplices, we will discuss it in the next section. It is indeed a special case of Theorem 3 below. We want find the conditions on the given matrices $D_{i}$ and $V_{i}$ for $f$ to be continuous on the domain. We first introduce the notations which will be used for the rest of this paper. Let $Q(a, b, c, d)$ denote a simplex with vertices $a, b, c$, and $d$, and let $\overrightarrow{a_{i}}$ denote the vector from 0 to the point $a_{i}$ in $\mathbb{R}^{3}$.

For nonzero and distinct points $a_{1}, a_{2}, a_{3}, a_{4}, a_{5}$ in $\mathbb{R}^{3}$, let $Q_{1}=Q_{1}\left(0, a_{1}, a_{4}\right.$, $\left.a_{5}\right), Q_{2}=Q_{2}\left(0, a_{1}, a_{2}, a_{5}\right), Q_{3}=Q_{3}\left(0, a_{2}, a_{3}, a_{5}\right)$, and $Q_{4}=Q_{4}\left(0, a_{3}, a_{4}, a_{5}\right)$. Note that $Q_{1}, Q_{2}, Q_{3}$, and $Q_{4}$ share a common edge $\overrightarrow{a_{5}}$. Furthermore, for $k \in\{1,2,3,4\}, T_{k}=\operatorname{span}\left\{\overrightarrow{a_{k}}, \overrightarrow{a_{5}}\right\}$ is the common plane shared by $Q_{k}$ and $Q_{k+1}$, except for $T_{4}$ which is the common plane shared by $Q_{4}$ and $Q_{1}$. Let $f_{i}(x)=A_{i} x$ be an affine mapping on $Q_{i}$ and $U_{i} D_{i} V_{i}^{T}$ be the decomposition of $A_{i}$. For $i, j \in\{1,2,3,4\}$, let

$$
X_{i}=D_{i} V_{i}^{T} \overrightarrow{a_{5}} \text { and } Y_{i j}=D_{i} V_{i}^{T} \overrightarrow{a_{j}} .
$$

The following theorem gives the necessary and sufficient condition for the function $f$ defined on $\bigcup_{i=1}^{4} Q_{i}$ and satisfying $f \mid Q_{i}=f_{i}$ to be a continuous function in $\bigcup_{i=1}^{4} Q_{i}$.

Theorem 2. For $1 \leq i \leq 4$, let an orthogonal matrix $V_{i}$ and a diagonal matrix $D_{i}$ with positive diagonal entries be given. The following two statements are equivalent.

(i) There exist orthogonal matrices $U_{1}, U_{2}, U_{3}$, and $U_{4}$ such that the function $f$ defined by $f \mid Q_{i}=f_{i}$ is continuous in $\bigcup_{i=1}^{4} Q_{i}$, where $f_{i}(x)=A_{i} x=U_{i} D_{i} V_{i}^{T} x$ for all $x$ in $Q_{i}$.

(ii) The matrices $D_{i}$ and $V_{i}$ satisfy the following face conditions : for $k=$ $1,2,3$

$$
\begin{aligned}
& x^{T} V_{k} D_{k}^{2} V_{k}^{T} y=x^{T} V_{k+1} D_{k+1}^{2} V_{k+1}^{T} y \quad \text { for all } x, y \in T_{k}, \\
& x^{T} V_{1} D_{1}^{2} V_{1}^{T} y=x^{T} V_{4} D_{4}^{2} V_{4}^{T} y \quad \text { for all } x, y \in T_{4},
\end{aligned}
$$

and two edge conditions :

$$
\begin{aligned}
&\left|X_{2} \times Y_{22}\right|^{2}\left|X_{3} \times Y_{33}\right|^{2}\left\{\left|X_{1}\right|^{2}\left(Y_{11} \cdot Y_{14}\right)-\left(X_{1} \cdot Y_{11}\right)\left(X_{1} \cdot Y_{14}\right)\right\} \\
&=\left\{\left|X_{2}\right|^{2}\left(Y_{21} \cdot Y_{22}\right)-\left(X_{2} \cdot Y_{21}\right)\left(X_{2} \cdot Y_{22}\right)\right\}\left\{\left\{\left|X_{3}\right|^{2}\left(Y_{32} \cdot Y_{33}\right)\right.\right. \\
&\left.-\left(X_{3} \cdot Y_{32}\right)\left(X_{3} \cdot Y_{33}\right)\right\}\left\{\left|X_{4}\right|^{2}\left(Y_{43} \cdot Y_{44}\right)-\left(X_{4} \cdot Y_{43}\right)\left(X_{4} \cdot Y_{44}\right)\right\}
\end{aligned}
$$




$$
\begin{aligned}
& \left.-\left|X_{3}\right|^{2}\left(X_{3} \times Y_{32} \cdot Y_{33}\right)\left(X_{4} \times Y_{43} \cdot Y_{44}\right)\right\} \\
& -\left|X_{2}\right|^{2}\left(X_{2} \times Y_{21} \cdot Y_{22}\right)\left\{( X _ { 3 } \times Y _ { 3 2 } \cdot Y _ { 3 3 } ) \left\{\left|X_{4}\right|^{2}\left(Y_{43} \cdot Y_{44}\right)\right.\right. \\
& \left.-\left(X_{4} \cdot Y_{43}\right)\left(X_{4} \cdot Y_{44}\right)\right\}+\left(X_{4} \times Y_{43} \cdot Y_{44}\right)\left\{\left|X_{3}\right|^{2}\left(Y_{32} \cdot Y_{33}\right)\right. \\
& \left.\left.-\left(X_{3} \cdot Y_{32}\right)\left(X_{3} \cdot Y_{33}\right)\right\}\right\}
\end{aligned}
$$

and

$$
\begin{aligned}
\mid X_{2} & \times\left. Y_{22}\right|^{2}\left|X_{3} \times Y_{33}\right|^{2}\left(X_{1} \times Y_{11} \cdot Y_{14}\right) \\
= & \left\{\left|X_{4}\right|^{2}\left(Y_{43} \cdot Y_{44}\right)-\left(X_{4} \cdot Y_{43}\right)\left(X_{4} \cdot Y_{44}\right)\right\}\left\{\left|X_{3}\right|^{2}\left(Y_{32} \cdot Y_{33}\right)\right. \\
& \left.-\left(X_{3} \cdot Y_{32}\right)\left(X_{3} \cdot Y_{33}\right)\right\}\left(X_{2} \times Y_{21} \cdot Y_{22}\right) \\
& +\left\{\left|X_{2}\right|^{2}\left(Y_{21} \cdot Y_{22}\right)-\left(X_{2} \cdot Y_{21}\right)\left(X_{2} \cdot Y_{22}\right)\right\}\left\{\left|X_{4}\right|^{2}\left(Y_{43} \cdot Y_{44}\right)\right. \\
& \left.-\left(X_{4} \cdot Y_{43}\right)\left(X_{4} \cdot Y_{44}\right)\right\}\left(X_{3} \times Y_{32} \cdot Y_{33}\right) \\
& +\left\{\left|X_{2}\right|^{2}\left(Y_{21} \cdot Y_{22}\right)-\left(X_{2} \cdot Y_{21}\right)\left(X_{2} \cdot Y_{22}\right)\right\}\left\{\left(\left|X_{3}\right|^{2}\left(Y_{32} \cdot Y_{33}\right)\right.\right. \\
& \left.-\left(X_{3} \cdot Y_{32}\right)\left(X_{3} \cdot Y_{33}\right)\right\}\left(X_{4} \times Y_{43} \cdot Y_{44}\right) \\
& -\left|X_{3}\right|^{2}\left(X_{2} \times Y_{21} \cdot Y_{22}\right)\left(X_{3} \times Y_{32} \cdot Y_{33}\right)\left(X_{4} \times Y_{43} \cdot Y_{44}\right) .
\end{aligned}
$$

Proof. Suppose that (i) holds. By Theorem 1, we obtain (2) and (3). It remains to be showed that the matrices $D_{i}$ and $V_{i}$ satisfy the edge conditions. From (2), we obtain

$$
X_{1} \cdot Y_{11}=\vec{a}_{5}^{T} V_{1} D_{1}^{2} V_{1}^{T} \vec{a}_{1}=\vec{a}_{5}^{T} V_{2} D_{2}^{2} V_{2}^{T} \vec{a}_{1}=X_{2} \cdot Y_{21} .
$$

Similarly, we have

$$
\begin{gathered}
X_{2} \cdot Y_{22}=X_{3} \cdot Y_{32}, \quad X_{4} \cdot Y_{44}=X_{1} \cdot Y_{14}, \quad X_{3} \cdot Y_{33}=X_{4} \cdot Y_{43} \\
\left|Y_{11}\right|^{2}=\left|Y_{21}\right|^{2}, \quad\left|Y_{22}\right|^{2}=\left|Y_{32}\right|^{2}, \quad\left|Y_{33}\right|^{2}=\left|Y_{43}\right|^{2}, \quad\left|Y_{44}\right|^{2}=\left|Y_{14}\right|^{2}
\end{gathered}
$$

and $\left|X_{1}\right|^{2}=\left|X_{2}\right|^{2}=\left|X_{3}\right|^{2}=\left|X_{4}\right|^{2}$.

If $B=\left[\begin{array}{ll}b_{1} & b_{2}\end{array}\right]$ is a $3 \times 2$ matrix, where $b_{1}$ and $b_{2}$ are column vectors, let $\widetilde{B}$ denote the $3 \times 3$ matrix $\left[\begin{array}{lll}b_{1} & b_{2} & \frac{b_{1} \times b_{2}}{\left|b_{1} \times b_{2}\right|}\end{array}\right]$.

Let $c_{j}=\left[\begin{array}{ll}\overrightarrow{a_{5}} & \overrightarrow{a_{j}}\end{array}\right]$. Then we have $D_{i} V_{i}^{T} c_{j}=\left[\begin{array}{ll}X_{i} & Y_{i j}\end{array}\right]$ and

$$
\widetilde{D_{i} V_{i}^{T} c_{j}}=\left[\begin{array}{lll}
X_{i} & Y_{i j} & \frac{X_{i} \times Y_{i j}}{\mid X_{i} \times Y_{i j}}
\end{array}\right] \text {. }
$$

Note that the inverse matrix of $\widetilde{D_{i} V_{i}^{T}} c_{j}$ is

$$
\left(\widetilde{D_{i} V_{i}^{T}} c_{j}\right)^{-1}=\frac{1}{\left|X_{i} \times Y_{i j}\right|^{2}}\left[\begin{array}{c}
\left(Y_{i j} \times\left(X_{i} \times Y_{i j}\right)\right)^{T} \\
\left(\left(X_{i} \times Y_{i j}\right) \times X_{i}\right)^{T} \\
\left|X_{i} \times Y_{i j}\right|\left(X_{i} \times Y_{i j}\right)^{T}
\end{array}\right] .
$$


Without loss of generality, we may assume that $U_{1}=I$. Since $U_{1} D_{1} V_{1}^{T} x=$ $U_{2} D_{2} V_{2}^{T} x$ for all $x \in T_{1}$, we have

$$
U_{2}=\widetilde{D_{1} V_{1}^{T}} c_{1}\left(\widetilde{D_{2} V_{2}^{T}} c_{1}\right)^{-1}
$$

Similarly, we obtain

$$
\begin{aligned}
& U_{3}=\left(\widetilde{U_{2}} \widetilde{D_{2} V_{2}^{T}} c_{2}\right)\left(\widetilde{D_{3} V_{3}^{T} c_{2}}\right)^{-1}, \\
& U_{4}=\left(\widetilde{U_{3}} \widetilde{D_{3} V_{3}^{T}} c_{3}\right)\left(\widetilde{D_{4} V_{4}^{T}} c_{3}\right)^{-1} \text {. }
\end{aligned}
$$

Let $W=U_{3} D_{3} V_{3}^{T} c_{3}$. From (4) and (5), we get

$$
W=\left(\widetilde{D_{1} V_{1}^{T} c_{1}}\right)\left(\widetilde{D_{2} V_{2}^{T} c_{1}}\right)^{-1}\left(\widetilde{D_{2} V_{2}^{T} c_{2}}\right)\left(\widetilde{D_{3} V_{3}^{T} c_{2}}\right)^{-1} D_{3} V_{3}^{T} c_{3} .
$$

Since $U_{1} D_{1} V_{1}^{T} x=U_{4} D_{4} V_{4}^{T} x$ for all $x \in T_{4}$, from (6) we obtain

$$
D_{1} V_{1}^{T} c_{4}=U_{4} D_{4} V_{4}^{T} c_{4}=\widetilde{W} P
$$

where $P=\left(\widetilde{D_{4} V_{4}^{T}} c_{3}\right)^{-1} D_{4} V_{4}^{T} c_{4}$. The equation (7) gives a relation between $D_{i}$ and $V_{i}$, which leads to the edge conditions of the part (ii). Let

$$
S=\left(\widetilde{D_{1} V_{1}^{T} c_{1}}\right)\left(\widetilde{D_{2} V_{2}^{T}} c_{1}\right)^{-1} D_{2} V_{2}^{T} c_{2} \text { and } R=\left(\widetilde{D_{3} V_{3}^{T} c_{2}}\right)^{-1} \widetilde{D_{3} V_{3}^{T} c_{3}} .
$$

Notice that $\widetilde{W}=\widetilde{S} R$. Next we want to simplify the matrices $S$ and $R$. We have

$$
\begin{aligned}
& S=\left[\begin{array}{lll}
X_{1} & Y_{11} & \left.\frac{X_{1} \times Y_{11}}{\mid X_{1} \times Y_{11}}\right]
\end{array}\right] \frac{1}{\left|X_{2} \times Y_{21}\right|^{2}}\left[\begin{array}{c}
\left(\left(Y_{21} \times\left(X_{2} \times Y_{21}\right)\right)^{T}\right. \\
\left(\left(X_{2} \times Y_{21}\right) \times X_{2}\right)^{T} \\
\left|X_{2} \times Y_{21}\right|\left(X_{2} \times Y_{21}\right)^{T}
\end{array}\right]\left[\begin{array}{ll}
X_{2} & Y_{22}
\end{array}\right] \\
& =\frac{1}{\left|X_{2} \times Y_{21}\right|^{2}}\left[\begin{array}{lll}
X_{1} & Y_{11} & \frac{X_{1} \times Y_{11}}{\left|X_{1} \times Y_{11}\right|}
\end{array}\right]\left[\begin{array}{cc}
\alpha_{1} & \alpha_{4} \\
0 & \alpha_{5} \\
0 & \alpha_{6}
\end{array}\right] \\
& =\frac{1}{\left|X_{2} \times Y_{21}\right|^{2}}\left[\begin{array}{ll}
\alpha_{1} X_{1} & \alpha_{4} X_{1}+\alpha_{5} Y_{11}+\frac{\alpha_{6}}{\left|X_{1} \times Y_{11}\right|} X_{1} \times Y_{11}
\end{array}\right] \text {, }
\end{aligned}
$$

where $\alpha_{1}=\left(Y_{21} \times\left(X_{2} \times Y_{21}\right)\right) \cdot X_{2}=\left|X_{2} \times Y_{21}\right|^{2}=\left|X_{1} \times Y_{11}\right|^{2}$,

$$
\alpha_{4}=\left(Y_{21} \times\left(X_{2} \times Y_{21}\right)\right) \cdot Y_{22}=\left|Y_{21}\right|^{2}\left(X_{2} \cdot Y_{22}\right)-\left(X_{2} \cdot Y_{21}\right)\left(Y_{21} \cdot Y_{22}\right) \text {, }
$$




$$
\begin{aligned}
& \alpha_{5}=\left(\left(X_{2} \times Y_{21}\right) \times X_{2}\right) \cdot Y_{22}=\left|X_{2}\right|^{2}\left(Y_{21} \cdot Y_{22}\right)-\left(X_{2} \cdot Y_{21}\right)\left(X_{2} \cdot Y_{22}\right), \\
& \alpha_{6}=\left|X_{2} \times Y_{21}\right|\left(X_{2} \times Y_{21} \cdot Y_{22}\right) .
\end{aligned}
$$

Hence,

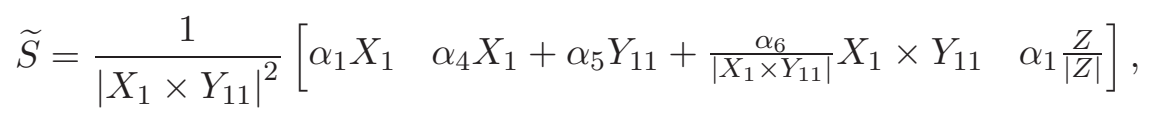

where

$$
Z=\frac{\alpha_{6}\left(X_{1} \cdot Y_{11}\right)}{\left|X_{1} \times Y_{11}\right|} X_{1}-\frac{\alpha_{6}\left|X_{1}\right|^{2}}{\left|X_{1} \times Y_{11}\right|} Y_{11}+\alpha_{5}\left(X_{1} \times Y_{11}\right)
$$

and

$$
|Z|=\left|X_{1} \times Y_{11}\right|^{2}\left|X_{2} \times Y_{22}\right|
$$

Note that

$$
\begin{aligned}
\alpha_{1} \frac{Z}{|Z|}= & \frac{\alpha_{6}\left(X_{1} \cdot Y_{11}\right)}{\left|X_{1} \times Y_{11}\right|\left|X_{2} \times Y_{22}\right|} X_{1}-\frac{\alpha_{6}\left|X_{1}\right|^{2}}{\left|X_{1} \times Y_{11}\right|\left|X_{2} \times Y_{22}\right|} Y_{11} \\
& +\frac{\alpha_{5}}{\left|X_{2} \times Y_{22}\right|}\left(X_{1} \times Y_{11}\right) .
\end{aligned}
$$

We also get

$$
\begin{aligned}
& R=\frac{1}{\left|X_{3} \times Y_{32}\right|^{2}}\left[\begin{array}{c}
\left(Y_{32} \times\left(X_{3} \times Y_{32}\right)\right)^{T} \\
\left(\left(X_{3} \times Y_{32}\right) \times X_{3}\right)^{T} \\
\left|X_{3} \times Y_{32}\right|\left(X_{3} \times Y_{32}\right)^{T}
\end{array}\right]\left[\begin{array}{lll}
X_{3} & Y_{33} & \frac{X_{3} \times Y_{33}}{\left|X_{3} \times Y_{33}\right|}
\end{array}\right] \\
& =\frac{1}{\left|X_{2} \times Y_{22}\right|^{2}}\left[\begin{array}{ccc}
\gamma_{1} & \gamma_{4} & \gamma_{7} \\
0 & \gamma_{5} & \gamma_{8} \\
0 & \gamma_{6} & \gamma_{9}
\end{array}\right] \text {, }
\end{aligned}
$$

where

$$
\begin{aligned}
& \gamma_{1}=\left(Y_{32} \times\left(X_{3} \times Y_{32}\right)\right) \cdot X_{3}=\left|X_{3} \times Y_{32}\right|^{2}=\left|X_{2} \times Y_{22}\right|^{2} \\
& \gamma_{4}=\left(Y_{32} \times\left(X_{3} \times Y_{32}\right)\right) \cdot Y_{33}=\left|Y_{32}\right|^{2}\left(X_{3} \cdot Y_{33}\right)-\left(X_{3} \cdot Y_{32}\right)\left(Y_{32} \cdot Y_{33}\right) \\
& \gamma_{5}=\left(\left(X_{3} \times Y_{32}\right) \times X_{3}\right) \cdot Y_{33}=\left|X_{3}\right|^{2}\left(Y_{32} \cdot Y_{33}\right)-\left(X_{3} \cdot Y_{32}\right)\left(X_{3} \cdot Y_{33}\right) \\
& \gamma_{6}=\left|X_{3} \times Y_{32}\right|\left(X_{3} \times Y_{32} \cdot Y_{33}\right) \\
& \gamma_{7}=\left(Y_{32} \times\left(X_{3} \times Y_{32}\right)\right) \cdot \frac{\left(X_{3} \times Y_{33}\right)}{\left|\left(X_{3} \times Y_{33}\right)\right|}=\frac{\left(X_{3} \cdot Y_{32}\right)}{\left|X_{3} \times Y_{33}\right|}\left(X_{3} \times Y_{32} \cdot Y_{33}\right) \\
& \gamma_{8}=\left(\left(X_{3} \times Y_{32}\right) \times X_{3}\right) \cdot \frac{\left(X_{3} \times Y_{33}\right)}{\left|\left(X_{3} \times Y_{33}\right)\right|}=-\frac{\left|X_{3}\right|^{2}}{\left|X_{3} \times Y_{33}\right|}\left(X_{3} \times Y_{32} \cdot Y_{33}\right), \\
& \gamma_{9}=\left|X_{3} \times Y_{32}\right|\left(X_{3} \times Y_{32}\right) \cdot \frac{\left(X_{3} \times Y_{33}\right)}{\left|\left(X_{3} \times Y_{33}\right)\right|}
\end{aligned}
$$




$$
=\frac{\left|X_{3} \times Y_{32}\right|}{\left|X_{3} \times Y_{33}\right|}\left(\left|X_{3}\right|^{2}\left(Y_{32} \cdot Y_{33}\right)-\left(X_{3} \cdot Y_{32}\right)\left(X_{3} \cdot Y_{33}\right)\right) .
$$

Therefore

$$
\begin{aligned}
\mid X_{1} & \times\left. Y_{11}\right|^{2}\left|X_{2} \times Y_{22}\right|^{2} \widetilde{W}=\left|X_{1} \times Y_{11}\right|^{2}\left|X_{2} \times Y_{22}\right|^{2} \widetilde{S} R \\
& =\left[\begin{array}{lll}
\alpha_{1} X_{1} & \alpha_{4} X_{1}+\alpha_{5} Y_{11}+\frac{\alpha_{6}}{\left|X_{1} \times Y_{11}\right|} X_{1} \times Y_{11} & \alpha_{1} \frac{Z}{|Z|}
\end{array}\right]\left[\begin{array}{ccc}
\gamma_{1} & \gamma_{4} & \gamma_{7} \\
0 & \gamma_{5} & \gamma 8 \\
0 & \gamma_{6} & \gamma_{9}
\end{array}\right] \\
& =\left[\begin{array}{lll}
\alpha_{1} \gamma_{1} X_{1} & \gamma_{4} F_{1}+\gamma_{5} F_{2}+\gamma_{6} F_{3} & \gamma_{7} F_{1}+\gamma_{8} F_{2}+\gamma_{9} F_{3}
\end{array}\right],
\end{aligned}
$$

where $F_{1}=\alpha_{1} X_{1}, F_{2}=\alpha_{4} X_{1}+\alpha_{5} Y_{11}+\frac{\alpha_{6}}{\left|X_{1} \times Y_{11}\right|} X_{1} \times Y_{11}$, and $F_{3}=\alpha_{1} \frac{Z}{|Z|}$. Note that

$$
P=\frac{1}{\left|X_{4} \times Y_{43}\right|^{2}}\left[\begin{array}{c}
\left(Y_{43} \times\left(X_{4} \times Y_{43}\right)\right)^{T} \\
\left(\left(X_{4} \times Y_{43}\right) \times X_{4}\right)^{T} \\
\left|X_{4} \times Y_{43}\right|\left(X_{4} \times Y_{43}\right)^{T}
\end{array}\right]\left[\begin{array}{ll}
X_{4} & Y_{44}
\end{array}\right]=\frac{1}{\left|X_{3} \times Y_{33}\right|^{2}}\left[\begin{array}{cc}
\beta_{1} & \beta_{4} \\
0 & \beta_{5} \\
0 & \beta_{6}
\end{array}\right],
$$

where

$$
\begin{aligned}
& \beta_{1}=\left(Y_{43} \times\left(X_{4} \times Y_{43}\right)\right) \cdot X_{4}=\left|X_{4} \times Y_{43}\right|^{2}=\left|X_{3} \times Y_{33}\right|^{2}, \\
& \beta_{4}=\left(Y_{43} \times\left(X_{4} \times Y_{43}\right)\right) \cdot Y_{44}=\left|Y_{43}\right|^{2}\left(X_{4} \cdot Y_{44}\right)-\left(X_{4} \cdot Y_{43}\right)\left(Y_{43} \cdot Y_{44}\right), \\
& \beta_{5}=\left(\left(X_{4} \times Y_{43}\right) \times X_{4}\right) \cdot Y_{44}=\left|X_{4}\right|^{2}\left(Y_{43} \cdot Y_{44}\right)-\left(X_{4} \cdot Y_{43}\right)\left(X_{4} \cdot Y_{44}\right), \\
& \beta_{6}=\left|X_{4} \times Y_{43}\right|\left(X_{4} \times Y_{43} \cdot Y_{44}\right) .
\end{aligned}
$$

Since $\alpha_{1}=\left|X_{1} \times Y_{11}\right|^{2}, \gamma_{1}=\left|X_{2} \times Y_{22}\right|^{2}$, and $\beta_{1}=\left|X_{3} \times Y_{33}\right|^{2}$, by (8) we obtain

$$
\alpha_{1} \gamma_{1} \beta_{1} \widetilde{W} P=\left[\begin{array}{ll}
\beta_{1} \alpha_{1} \gamma_{1} X_{1} & \beta_{4} B_{1}+\beta_{5} B_{2}+\beta_{6} B_{3}
\end{array}\right],
$$

where $B_{1}=\alpha_{1} \gamma_{1} X_{1}, B_{2}=\gamma_{4} F_{1}+\gamma_{5} F_{2}+\gamma_{6} F_{3}$, and $B_{3}=\gamma_{7} F_{1}+\gamma_{8} F_{2}+\gamma_{9} F_{3}$. Since, by (7), $\left[\begin{array}{ll}X_{1} & Y_{14}\end{array}\right]=D_{1} V_{1}^{T} c_{4}=U_{4} D_{4} V_{4}^{T} c_{4}=\widetilde{W} P$, we have

$$
\alpha_{1} \beta_{1} \gamma_{1} Y_{14}=\beta_{4} B_{1}+\beta_{5} B_{2}+\beta_{6} B_{3} .
$$

Since $\left\{X_{1}, Y_{11}, X_{1} \times Y_{11}\right\}$ is a basis for $\mathbb{R}^{3}$, we can write $Y_{14}$ uniquely as a linear combination of the elements of this basis. Indeed

$$
Y_{14}=\frac{X_{1} \times Y_{11} \cdot Y_{14}}{\left|X_{1} \times Y_{11}\right|^{2}}\left(X_{1} \times Y_{11}\right)+\alpha X_{1}+\beta Y_{11},
$$

where

$$
\alpha=\frac{1}{\left|X_{1}\right|^{2}\left|Y_{11}\right|^{2}-\left(X_{1} \cdot Y_{11}\right)^{2}}\left(\left|Y_{11}\right|^{2}\left(X_{1} \cdot Y_{14}\right)-\left(X_{1} \cdot Y_{11}\right)\left(Y_{11} \cdot Y_{14}\right)\right)
$$


and

$$
\beta=\frac{1}{\left|X_{1}\right|^{2}\left|Y_{11}\right|^{2}-\left(X_{1} \cdot Y_{11}\right)^{2}}\left(\left|X_{1}\right|^{2}\left(Y_{11} \cdot Y_{14}\right)-\left(X_{1} \cdot Y_{11}\right)\left(X_{1} \cdot Y_{14}\right)\right) .
$$

By substituting the values of $F_{1}, F_{2}$, and $F_{3}$ into $B_{1}, B_{2}$, and $B_{3}$, we obtain

$$
\begin{aligned}
\beta_{4} B_{1}+\beta_{5} B_{2} & +\beta_{6} B_{3} \\
= & \left\{\beta_{4} \gamma_{1} \alpha_{1}+\beta_{5} \gamma_{4} \alpha_{1}+\beta_{5} \gamma_{5} \alpha_{4}+\beta_{6} \gamma_{7} \alpha_{1}+\beta_{6} \gamma_{8} \alpha_{4}\right. \\
& \left.+\frac{\left(\beta_{5} \gamma_{6}+\beta_{6} \gamma_{9}\right) \alpha_{6}\left(X_{1} \cdot Y_{11}\right)}{\left|X_{1} \times Y_{11}\right|\left|X_{2} \times Y_{22}\right|}\right\} X_{1} \\
& +\left(\beta_{5} \gamma_{5} \alpha_{5}+\beta_{6} \gamma_{8} \alpha_{5}-\frac{\left(\beta_{5} \gamma_{6}+\beta_{6} \gamma_{9}\right) \alpha_{6}\left|X_{1}\right|^{2}}{\left|X_{1} \times Y_{11}\right|\left|X_{2} \times Y_{22}\right|}\right) Y_{11} \\
& +\left(\frac{\left(\beta_{5} \gamma_{5}+\beta_{6} \gamma_{8}\right) \alpha_{6}}{\left|X_{1} \times Y_{11}\right|}+\frac{\left(\beta_{5} \gamma_{6}+\beta_{6} \gamma_{9}\right) \alpha_{5}}{\left|X_{2} \times Y_{22}\right|}\right)\left(X_{1} \times Y_{11}\right) .
\end{aligned}
$$

After multiplying (10) by $\alpha_{1} \beta_{1} \gamma_{1}$ and comparing the coefficients of $Y_{11}$ and also the coefficients of $X_{1} \times Y_{11}$, we obtain

$$
\begin{aligned}
\left|X_{2} \times Y_{22}\right|^{2}\left|X_{3} \times Y_{33}\right|^{2}\left(\left|X_{1}\right|^{2}\right. & \left.\left(Y_{11} \cdot Y_{14}\right)-\left(X_{1} \cdot Y_{11}\right)\left(X_{1} \cdot Y_{14}\right)\right) \\
& =\beta_{5} \gamma_{5} \alpha_{5}+\beta_{6} \gamma_{8} \alpha_{5}-\frac{\left(\beta_{5} \gamma_{6}+\beta_{6} \gamma_{9}\right) \alpha_{6}\left|X_{1}\right|^{2}}{\left|X_{1} \times Y_{11}\right|\left|X_{2} \times Y_{22}\right|}
\end{aligned}
$$

and

$$
\beta_{1} \gamma_{1}\left(X_{1} \times Y_{11} \cdot Y_{14}\right)=\frac{\left(\beta_{5} \gamma_{5}+\beta_{6} \gamma_{8}\right) \alpha_{6}}{\left|X_{1} \times Y_{11}\right|}+\frac{\left(\beta_{5} \gamma_{6}+\beta_{6} \gamma_{9}\right) \alpha_{5}}{\left|X_{2} \times Y_{22}\right|} .
$$

After substituting the values of $\alpha_{i}, \beta_{i}$, and $\gamma_{i}$, these two equations can be simplified into two edge conditions, respectively. This proves that (i) implies (ii), as needed.

Suppose next that (ii) holds. Let $U_{1}=I$. From (2) and Theorem 1, there exist orthogonal matrices $U_{2}, U_{3}$, and $U_{4}$ such that

$$
U_{k} D_{k} V_{k}^{T} x=U_{k+1} D_{k+1} V_{k+1}^{T} x, \text { for all } x \in T_{k},
$$

where $k=1,2,3$. Also from (3) and Theorem 1, there exists an orthogonal matrix $U_{4}^{*}$ such that

$$
D_{1} V_{1}^{T} w=U_{4}^{*} D_{4} V_{4}^{T} w, \text { for all } w \in T_{4} .
$$


Let $A_{i}=U_{i} D_{i} V_{i}^{T}$ for all $1 \leq i \leq 4$ and $A_{4}^{*}=U_{4}^{*} D_{4} V_{4}^{T}$. On each simplex $Q_{i}$, we define $f_{i}(x)=A_{i} x$. Furthermore, we also define $f_{4}^{*}(x)=A_{4}^{*} x$ for $x \in Q_{4}$.

Now we have the simplices $f_{1}\left(Q_{1}\right)$ and $f_{2}\left(Q_{2}\right)$ sharing a common face $f_{1}\left(T_{1}\right)=f_{2}\left(T_{1}\right)$, the simplices $f_{2}\left(Q_{2}\right)$ and $f_{3}\left(Q_{3}\right)$ sharing a common face $f_{2}\left(T_{2}\right)=f_{3}\left(T_{2}\right)$, the simplices $f_{3}\left(Q_{3}\right)$ and $f_{4}\left(Q_{4}\right)$ sharing a common face $f_{3}\left(T_{3}\right)=f_{4}\left(T_{3}\right)$, and the simplices $f_{1}\left(Q_{1}\right)$ and $f_{4}^{*}\left(Q_{4}\right)$ sharing a common face $f_{1}\left(T_{4}\right)=f_{4}^{*}\left(T_{4}\right)$. In order to obtain a continuous piecewise affine mapping $f$ on $\bigcup_{i=1}^{4} Q_{i}$ such that $f \mid Q_{i}=f_{i}$, we need to show that

$$
U_{4} D_{4} V_{4}^{T}\left(T_{4}\right)=U_{4}^{*} D_{4} V_{4}^{T}\left(T_{4}\right)=D_{1} V_{1}^{T}\left(T_{4}\right) .
$$

Let the angle $\theta_{1}$ be the angle between $f_{1}\left(T_{1}\right)$ and $f_{1}\left(T_{4}\right)$, the angle $\theta_{2}$ be the angle between $f_{2}\left(T_{1}\right)$ and $f_{2}\left(T_{2}\right), \theta_{3}$ be the angle between $f_{3}\left(T_{2}\right)$ and $f_{3}\left(T_{3}\right)$, and $\theta_{4}$ be the angle between $f_{4}\left(T_{3}\right)$ and $f_{4}\left(T_{4}\right)$. To achieve the equation (11), we only need to show that $\theta_{1}+\theta_{2}+\theta_{3}+\theta_{4}=2 \pi$. Since $\theta_{1}$ is the angle between $f_{1}\left(T_{1}\right)$ and $f_{1}\left(T_{4}\right)$, we have

$$
\begin{aligned}
\sin \theta_{1} & =\frac{\left|\left(X_{1} \times Y_{11}\right) \times\left(X_{1} \times Y_{14}\right)\right|}{\left|X_{1} \times Y_{11}\right|\left|X_{1} \times Y_{14}\right|}=\frac{\left|X_{1}\right|\left|X_{1} \times Y_{11} \cdot Y_{14}\right|}{\left|X_{1} \times Y_{11}\right|\left|X_{1} \times Y_{14}\right|} \quad \text { and } \\
\cos \theta_{1} & =\frac{\left(X_{1} \times Y_{11}\right) \cdot\left(X_{1} \times Y_{14}\right)}{\left|X_{1} \times Y_{11}\right|\left|X_{1} \times Y_{14}\right|} \\
& =\frac{\left|X_{1}\right|^{2}\left(Y_{11} \cdot Y_{14}\right)-\left(X_{1} \cdot Y_{11}\right)\left(X_{1} \cdot Y_{14}\right)}{\left|X_{1} \times Y_{11}\right|\left|X_{1} \times Y_{14}\right|} .
\end{aligned}
$$

Similarly, we obtain

$$
\begin{aligned}
\sin \theta_{2} & =\frac{\left|X_{2}\right|\left|X_{2} \times Y_{21} \cdot Y_{22}\right|}{\left|X_{2} \times Y_{21}\right|\left|X_{2} \times Y_{22}\right|}, \quad \sin \theta_{3}=\frac{\left|X_{3}\right|\left|X_{3} \times Y_{32} \cdot Y_{33}\right|}{\left|X_{3} \times Y_{32}\right|\left|X_{3} \times Y_{33}\right|} \\
\sin \theta_{4} & =\frac{\left|X_{4}\right|\left|X_{4} \times Y_{43} \cdot Y_{44}\right|}{\left|X_{4} \times Y_{43}\right|\left|X_{4} \times Y_{44}\right|}, \\
\cos \theta_{2} & =\frac{\left|X_{2}\right|^{2}\left(Y_{21} \cdot Y_{22}\right)-\left(X_{2} \cdot Y_{21}\right)\left(X_{2} \cdot Y_{22}\right)}{\left|X_{2} \times Y_{21}\right|\left|X_{2} \times Y_{22}\right|} \\
\cos \theta_{3} & =\frac{\left|X_{3}\right|^{2}\left(Y_{32} \cdot Y_{33}\right)-\left(X_{3} \cdot Y_{32}\right)\left(X_{3} \cdot Y_{33}\right)}{\left|X_{3} \times Y_{32}\right|\left|X_{3} \times Y_{33}\right|} \\
\cos \theta_{4} & =\frac{\left|X_{4}\right|^{2}\left(Y_{43} \cdot Y_{44}\right)-\left(X_{4} \cdot Y_{43}\right)\left(X_{4} \cdot Y_{44}\right)}{\left|X_{4} \times Y_{43}\right|\left|X_{4} \times Y_{44}\right|}
\end{aligned}
$$

Hence, from the first edge conditions, we obtain

$$
\cos \theta_{1}=\cos \theta_{2}\left(\cos \theta_{3} \cos \theta_{4}-\sin \theta_{3} \sin \theta_{4}\right)
$$




$$
\begin{aligned}
& -\sin \theta_{2}\left(\cos \theta_{3} \sin \theta_{4}+\sin \theta_{3} \cos \theta_{4}\right) \\
= & \cos \theta_{2}\left(\cos \left(\theta_{3}+\theta_{4}\right)\right)-\sin \theta_{2}\left(\sin \left(\theta_{3}+\theta_{4}\right)\right) \\
= & \cos \left(\theta_{2}+\theta_{3}+\theta_{4}\right)
\end{aligned}
$$

and, from the second edge condition,

$$
\begin{aligned}
-\sin \theta_{1}= & \sin \theta_{2}\left(\cos \theta_{3} \cos \theta_{4}+\sin \theta_{3} \sin \theta_{4}\right) \\
& +\cos \theta_{2}\left(\cos \theta_{3} \sin \theta_{4}+\sin \theta_{3} \cos \theta_{4}\right) \\
= & \sin \theta_{2}\left(\cos \left(\theta_{3}+\theta_{4}\right)\right)+\cos \theta_{2}\left(\sin \left(\theta_{3}+\theta_{4}\right)\right) \\
= & \sin \left(\theta_{2}+\theta_{3}+\theta_{4}\right) .
\end{aligned}
$$

It is readily verified that $\cos \left(\theta_{1}+\theta_{2}+\theta_{3}+\theta_{4}\right)=\cos ^{2} \theta_{1}+\sin ^{2} \theta_{1}=1$. Since $0<\theta_{1}+\theta_{2}+\theta_{3}+\theta_{4}<4 \pi$, we must have $\theta_{1}+\theta_{2}+\theta_{3}+\theta_{4}=2 \pi$, as desired. This completes the proof of Theorem 3 .

\section{Three Simplices Sharing a Common Edge}

In this section we will establish the analogous result to Theorem 3 for the case when a domain is a union of three simplices. In fact, Sinthaveelert already established a result for this case in [1]. Here we will induce the result by using Theorem 3. By using the same notations as in the previous section and considering $Q_{3}=Q_{4}$ and $A_{3}=A_{4}$, we get

$$
X_{3}=X_{4}, Y_{33}=Y_{43}=Y_{44}, \text { and } Y_{13}=Y_{14} .
$$

Then we derive the following theorem.

Theorem 3. For $1 \leq i \leq 3$, let an orthogonal matrix $V_{i}$ and a diagonal matrix $D_{i}$ with positive diagonal entries be given. The following two statements are equivalent.

(i) There exist orthogonal matrices $U_{1}, U_{2}$, and $U_{3}$ such that the function $f$ defined by $f \mid Q_{i}=f_{i}$ is continuous in $\bigcup_{i=1}^{3} Q_{i}$, where $f_{i}(x)=A_{i} x=U_{i} D_{i} V_{i}^{T} x$ for all $x$ in $Q_{i}$.

(ii) The matrices $D_{i}$ and $V_{i}$ satisfy the following face conditions : for $k=1,2$

$$
\begin{aligned}
& x^{T} V_{k} D_{k}^{2} V_{k}^{T} y=x^{T} V_{k+1} D_{k+1}^{2} V_{k+1}^{T} y \quad \text { for all } x, y \in T_{k}, \\
& x^{T} V_{1} D_{1}^{2} V_{1}^{T} y=x^{T} V_{3} D_{3}^{2} V_{3}^{T} y \quad \text { for all } x, y \in T_{3},
\end{aligned}
$$

and two edge conditions :

$$
\left|X_{2} \times Y_{22}\right|^{2}\left\{\left|X_{1}\right|^{2}\left(Y_{11} \cdot Y_{13}\right)-\left(X_{1} \cdot Y_{11}\right)\left(X_{1} \cdot Y_{13}\right)\right\}
$$




$$
\begin{aligned}
= & \left\{\left|X_{2}\right|^{2}\left(Y_{21} \cdot Y_{22}\right)-\left(X_{2} \cdot Y_{21}\right)\left(X_{2} \cdot Y_{22}\right)\right\}\left\{\left|X_{3}\right|^{2}\left(Y_{32} \cdot Y_{33}\right)\right. \\
& \left.-\left(X_{3} \cdot Y_{32}\right)\left(X_{3} \cdot Y_{33}\right)\right\}-\left|X_{2}\right|^{2}\left(X_{2} \times Y_{21} \cdot Y_{22}\right)\left(X_{3} \times Y_{32} \cdot Y_{33}\right)
\end{aligned}
$$

and

$$
\begin{aligned}
\left|X_{2} \times Y_{22}\right|^{2}\left(X_{1} \times Y_{11} \cdot Y_{13}\right) \\
=\left(X_{2} \times Y_{21} \cdot Y_{22}\right)\left\{\left|X_{3}\right|^{2}\left(Y_{32} \cdot Y_{33}\right)-\left(X_{3} \cdot Y_{32}\right)\left(X_{3} \cdot Y_{33}\right)\right\} \\
\quad+\left(X_{3} \times Y_{32} \cdot Y_{33}\right)\left\{\left|X_{2}\right|^{2}\left(Y_{21} \cdot Y_{22}\right)-\left(X_{2} \cdot Y_{21}\right)\left(X_{2} \cdot Y_{22}\right)\right\} .
\end{aligned}
$$

\section{References}

[1] Malinee Sinthaveelert, Prescribing Dilatations in Space, Ph.D. Thesis, University of Illinois at Urbana-Champaign, IL, USA (2008).

[2] D.C. Lay, Linear Algebra and its Applications, Second Edition, Addison Wesley Longman, USA (1996). 